\title{
COLECCION Y MANTENIMIENTO DE GERMOPLASMA DE ACHIOTE (Bixa orellana L.) EN LA AMAZONIA PERUANA
}

A gustín Gonzales Coral *

\section{RESUMEN}

En la A mazonía Peruana existe un gran variabilidad genética de la especie Bixa orellana L. "achiote", esta información se fundamenta en las 58 colecciones que se han realizado en diferentes lugares de la Selva Peruana, en las cuales se pueden observar una gran diversidad en lo que se refiere a: hábito de crecimiento (arbustos y árboles); coloración del tallo (gris, anaranjado y marrón); color de las hojas (verdes con diferentes tonalidades y violetas); color de las flores (blancas y violetas con diferentes tonalidades); color de los frutos (verdes con diferentes tonalidades; amarillo con diferentes tonalidades, anaranjado, rojo con diferentes tonalidades, marrón y negro); forma de los frutos (ovoide, redondo elíptico y cónico); espinosidad de los frutos (sin espinas, muy baja, baja, alta y muy alta espinosidad); longitud de las espinas (muy cortas, cortas, largas y muy largas); número de semillas por fruto, etc.

Todo este material se encuentra in vivo en un área de 2 ha, en el banco de germoplasma de achiote, ubicado en el campo experimental 'ALLPAHUAYO' del Instituto de Investigaciones de la A mazonía Peruana (IIAP), situado en el Km. 20.5 de la carretera Iquitos - $\mathrm{N}$ auta.

Palabras Claves: A mazonía Peruana, Germoplasma, achiote, Bixa orellana

\section{INTRODUCCION}

En la amazonía peruana, se cuenta con un gran potencial de recursos fitogenéticos, dentro de las cuales existe una amplia diversidad de especies, que podrían integrarse al sector agro rural; por éste motivo se ha seleccionado la especie Bixa orellana L. achiote" para realizar investigaciones que, en la primera fase comprende colección, mantenimiento, evaluación, caracterización, documentación e intercambio de material reproductivo.

El presente artículo está orientado a satisfacer los primeros pasos de la investigación (colección y mantenimiento), cuyo objetivo principal es de contar con

INSTITUTO DE INVESTIGACIONES DE LA AMAZONIA PERUANA IIAP Av. Abelardo Quiñones Km. 2.5 A partado 784 Iquitos - Perú 
mayor diversidad genética, para ingresar después al campo de la evaluación, caracterización y mejoramiento a fin de ofrecer al agro regional un producto que garantice el desarrollo agrícola.

La importancia que ha tomado realizar estudios de investigación, para el desarrollo con esta especie radica en que muchos aditivos o colorantes, tienen efectos cancerígenos, siendo la BIXINA, colorante natural obtenido de la semilla del A chiote, uno de los pocos autorizados para utilizarse en la alimentación natación por la Organización Mundial para la Salud (OMS), dado que no es tóxico, es insipido y no altera el sabor de los alimentos.

\section{MATERIAL Y METODOS}

\section{$2.1 \quad$ Materiales}

\subsubsection{Ubicación del banco de germoplasma}

El banco de germoplasma está ubicado en el campo experimental "A L PA HUAY O" en el Km. 20.5 de la carretera I quitos

- Nauta (figura 1) cuya altitud es de 126 m.s.n.m; siendo sus coordenadas geográficas las siguientes: Latitud 030, 450 y Longitud 750 15; con una extensión aproximada de dos ha.

Según la clasificación de Holdridge (1959), la ciudad de Iquitos está considerada como bosque húmedo tropical, caracterizado por presentar lluvias durante todo el año con precipitación que oscila entre 2,000 y 4,000 $\mathrm{m} \mathrm{m}$. Y una temperatura media anual de26.52C.EI suelo presenta una textura francoarcillo-arenoso con pH ácido y un contenido de materia orgánica media.

\subsection{Achiote (Bixa orellana L.)}

Según la clasificación botánica, la especie Bixa orellana pertenece a:

División

Sub División

Sección

Sub Sección

Clase

Sub Clase

Orden

Familia

Género

Especie
Embriofita

Diploidalia

Espermatofita

(Fanerógamas)

A ngiosperma

Dicotiledonea

A rquiclamidea

Parietales

Bixaceas

Bixa

Bixa orellana L. 


\section{FIGURA 1.- CROQUIS DE UBICACION DEL BANCO DE GERMOPLASMA} DE ACHIOTE

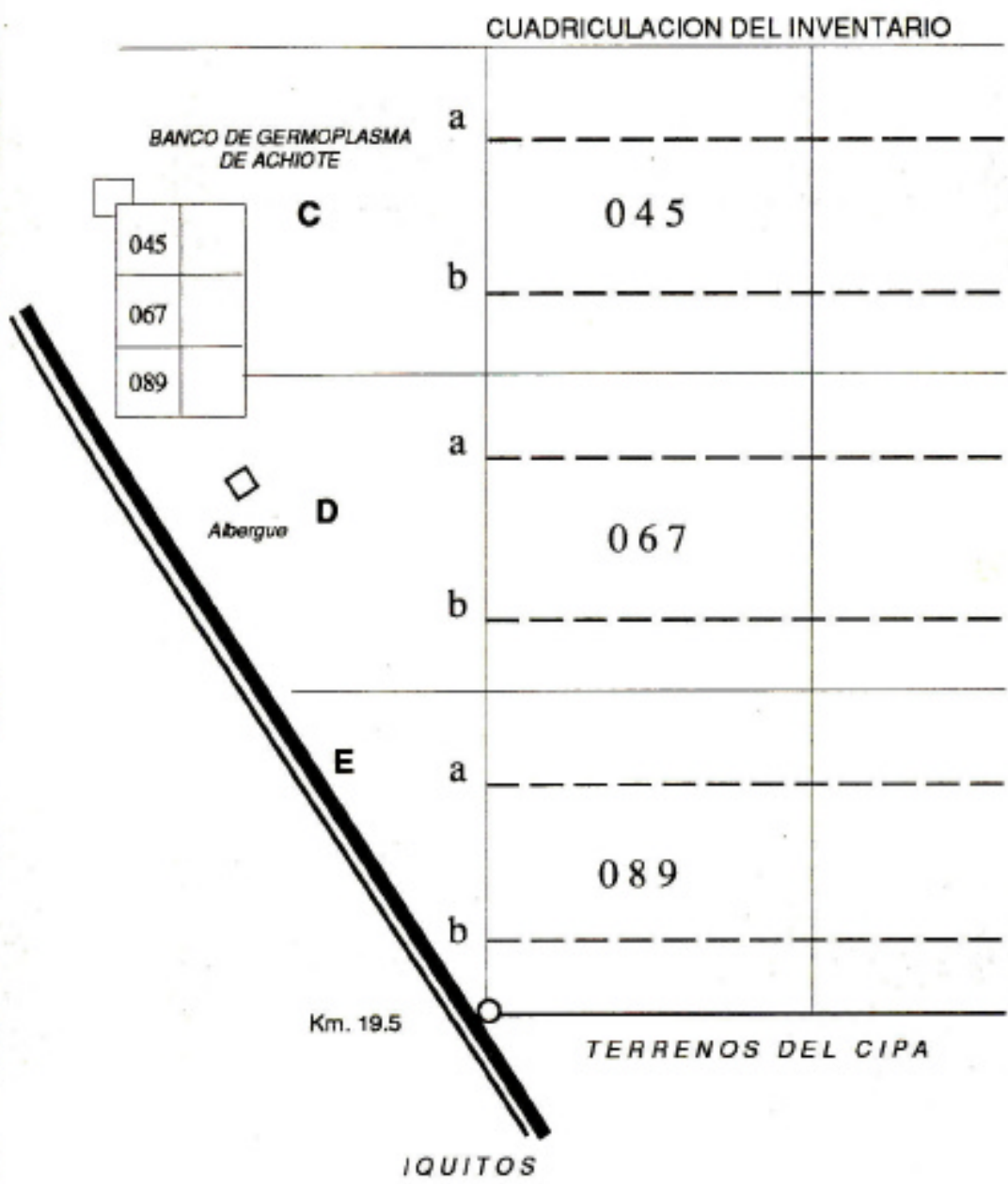

Fuande do informacion Cartogrtice Mape Bese del huenterio Forasta Alashuolo 1966 
La descripción de la especie reporta los siguientes:

Hábito.- Árbol bajo de copa redonda, excepcionalmente alcanza hasta los 10 metros de altura (León, 1968, citado por Nájar 1985), corteza pardo y el árbol presenta una forma muy variada, ramas delgadas tendiendo a leñosas y su coloración varia de verde a morado, ramificación dicotómica y se inicia desde la base del tronco.

Hojas.- Simples, alternas, ovadas, precio la dos y palabras en ambas caras, tiene de 8 a $10 \mathrm{~cm}$. de largo y de 4 a $15 \mathrm{~cm}$. de ancho, con un ápice acuminado, disminuyendo gradualmente y la base truncada es algo acorazonada, el color del envés es algo plateada especialmente cuando maduras, las mismas que se tornan algo coriáceas.

Flores. - Son panículas terminales, la floración es escalonada, la apertura de las flores ocurren primero en la posición superior y luego se abren de la parte inferior.

Las flores tienen 5 sépalos, caducos, rojizos o blancos, ovales que miden 2.4 a $2.8 \mathrm{~cm}$. de largo en el ápice: el color puede variar desde blanco hasta rosado o morado, el tamaño es variado, el bastón floral es globuloso, dando la impresión de estar recubierto por sucesivas capas (sépalos).

L as flores poseen 5 pétalos, redondos o de forma ovalada de 1 a $2 \mathrm{~cm}$. de largo, rosado o blancos según el cultivar. Los estambres son pequeños con pedúnculos cortos muy numerosos (350 -400), dispuestos al rededor del pistilo, la antera contiene 8 sacos embrionarios que producen abundante polen.

El pistilo tiene un estigma en forma de boca abierta y el estilo es alargado, erecto y llega a tener la forma de ' $S$ " al madurar, termina en un ovario de tipo elipsoidal supero recubierto por muchos pelos glándulares.

El ovario consta de 2 valvas, ocasionalmente 3, a cuyos costados en la placenta al berga numerosos óvulos.

$L$ as flores son hermafroditas, regulares, bisexuales y actinomórficos.

Fruto.- Es una cápsula dehisente, hemisferica, ovoide, elipscidalo cónica, cubierta por espinas largas y suaves en la mayoría de los cultivares, lisa en algunas mutantes y su color puede ser rojo, café, verde o amarillo cuando está maduro y se abren en dos valvas, las paredes son delgadas y en el lado interno de cada valva hay una placenta, que se prolonga en una membrana blanca adherida en parte a la pared del fruto, presenta placentación parietal laminar.

Cada placenta lleva numerosas semillas recubierta por una membrana fina y blancusca, debajo de esta hay una capa de parenquima acuoso anaranjado brillante o rojizo amarillento.

Se puede encontrar frutos trivalvares, lo cual puede ser una característica beneficiosa para aumentar la capacidad de producción. Del arilo de la semilla se obtiene la bixina que viene a ser un carotenoide, que es muy utilizado en industria alimentaría. 


\subsection{Metodología}

\subsubsection{Colecciones: Para realizar las colecciones fueron necesarios los siguientes pasos:}

a) Determinación de los límites de ocurrencia geográfica, esto se realizó básicamente para tener un conocimiento sobre el origen de la especie: según Hernández - (1988), la especie es originaria de A mérica Tropical, por esto se le ve crecer en toda la selva; según Burkhill, citado por Catalan (1974), la especie es originaria de la parte $\mathrm{N}$ orte de A mérica del Sur, de donde fue llevada a los países tropicales y sub tropicales de los hemisferios oriental y occidental; según Rodriguez (1983) el achiote es un árbol de A mérica tropical.

b) Confección de un mapa de distribución de la especie; con toda la información recopilada se confeccionó un mapa de distribución de la especie, con el cual se programó los viajes de colección (figura 2)

c) Viaje de reconocimiento, caracterización y colección de achiote; para este fin se realizaron viajes a las localidades de Tingo María y Pucallpa; en Loreto, se visitó localidades ubicadas aguas abajo del río A mazonas, río $\mathrm{N}$ apo, río $\mathrm{N}$ anay y poblados cercanos a la ciudad de Iquitos.

\subsubsection{Mantenimiento Con el fin de mantener las colecciones se realizaron las siguientes actividades:}

a) Preparación de plantones; se instalé un vivero, donde se prepararon las plantas hasta el momento óptimo de su traslado al campo definitivo, se opté por la técnica de preparación por semillas y siembra en bolsas (Ocampo, 1983), que consiste en emplear bolsas plásticas de $20 \mathrm{~cm}$. de diámetro por $30 \mathrm{~cm}$. de alto, preferentemente de color negro, para ser llenadas con tierra preparada de la siguiente manera: $30 \%$ de estiércol de aves y $70 \%$ de tierra, tratada con un fungicida: se deben colocar de 3-4 semillas, a una profundidad de $2 \mathrm{~cm}$. Cuando las plantas tienen $10 \mathrm{~cm}$. de altura se debe ralear, dejando solamente las más vigorosa, y esperar que la planta tenga aproximadamente $30 \mathrm{~cm}$. De alto, para su transplante al campo definitivo. 
b) Preparación del campo definitivo; se realizó las labores tradicionales de preparación del terreno (rozo, tumba, quema y limpieza). El trazado de campo se realizó teniendo en cuenta la densidad de plantas: $5 \mathrm{~m}$. entre accesos (filas) y4 $\mathrm{m}$. entre plantas, con hoyos de $40 \times 40 \times 30 \mathrm{~cm}$.

c) Labores culturales; la labor de mayor importancia, fue el control de las malas hierbas, para lo cual se realizó deshierbos manuales con un intervalo de 4 meses: no se realizó control de plagas y enfermedades porque la presencia no fue significativa. 


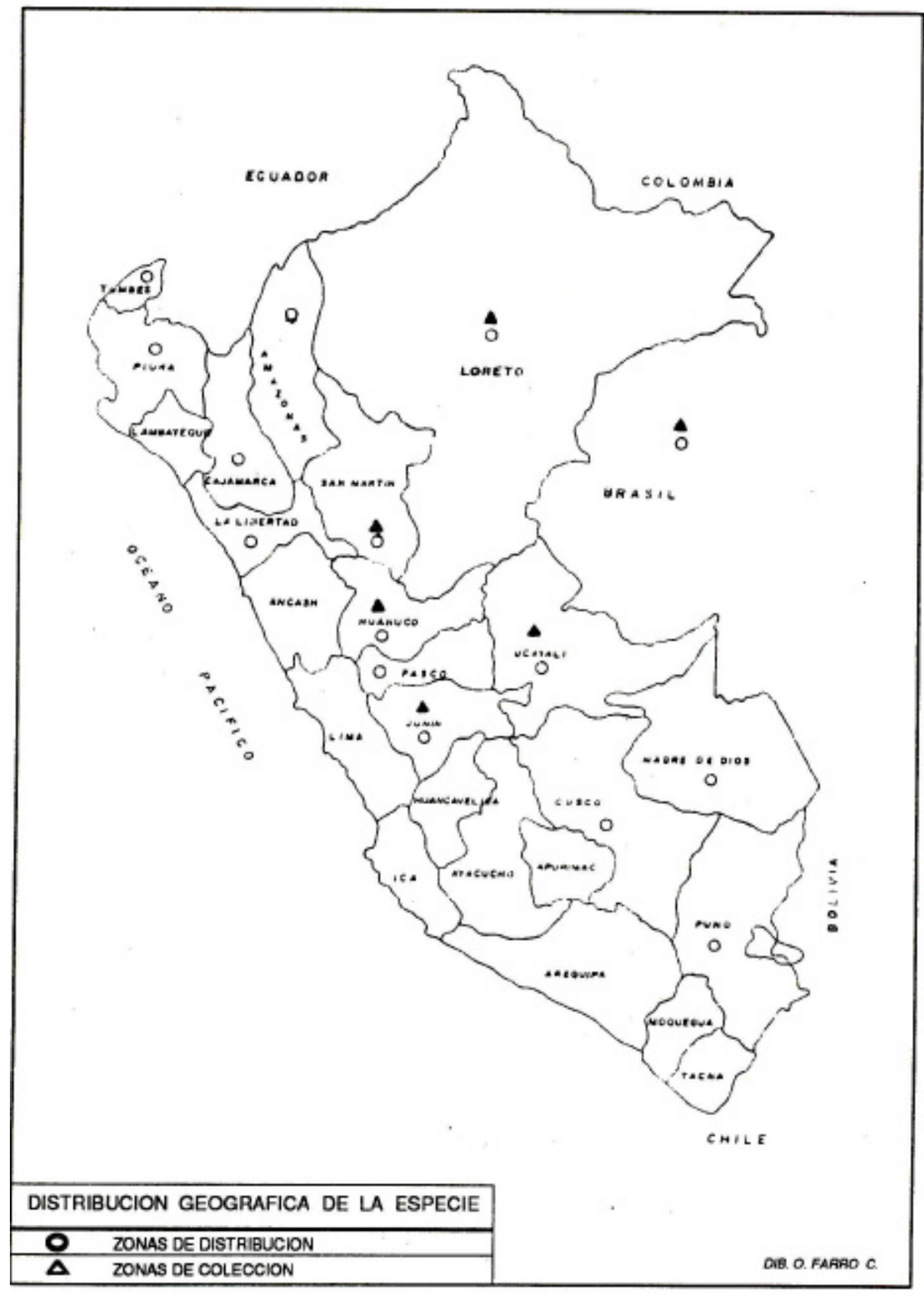




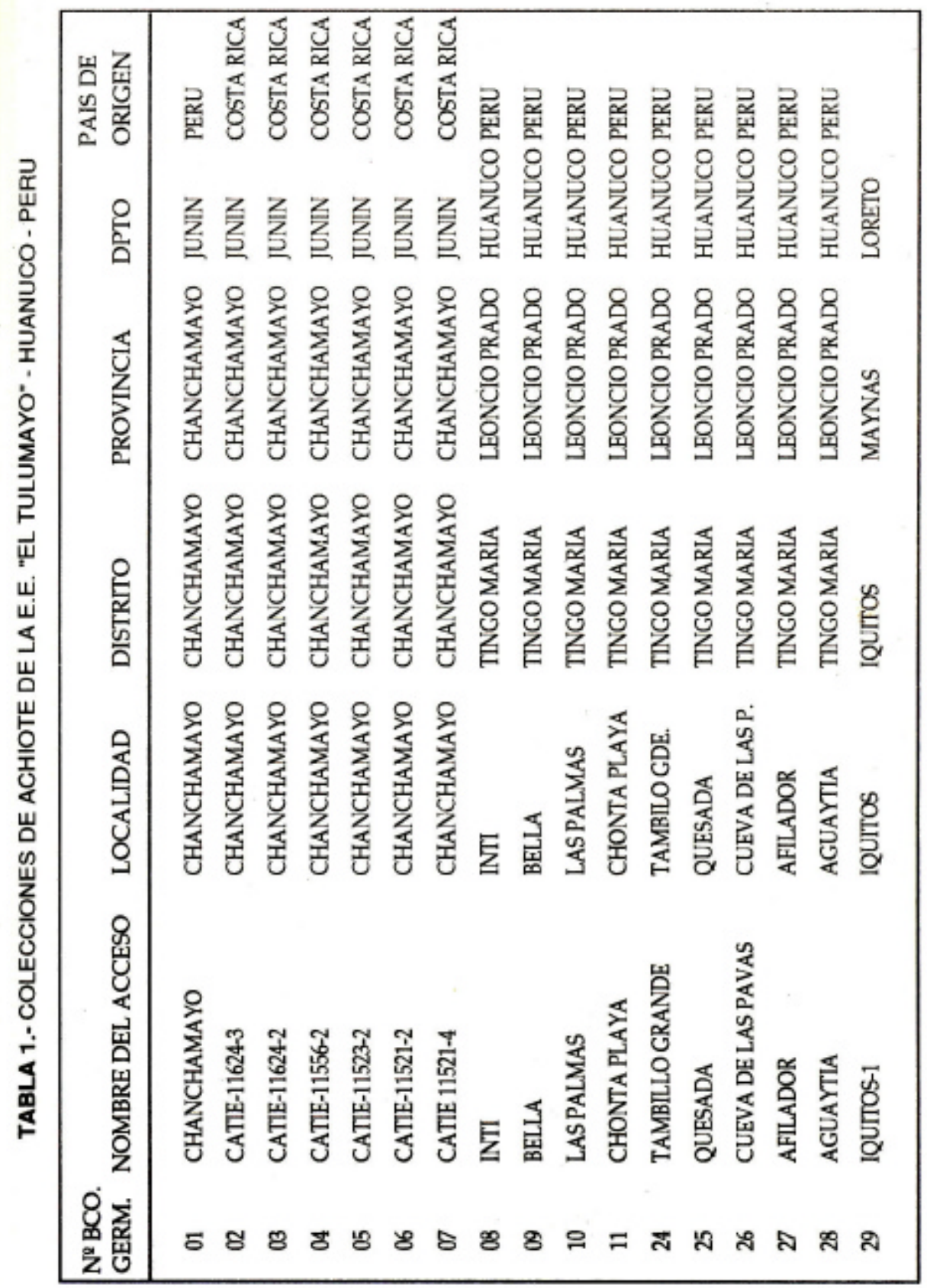




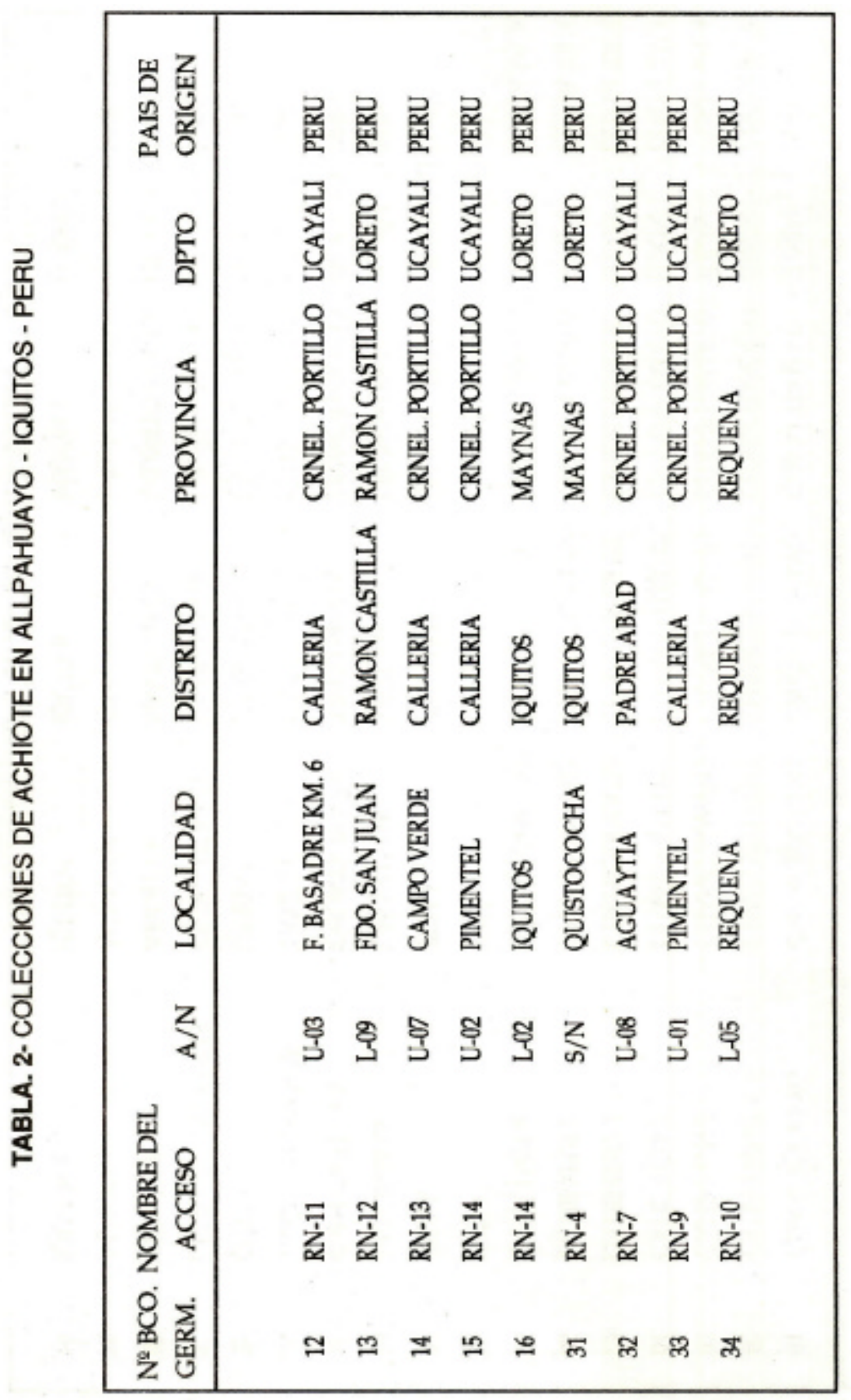

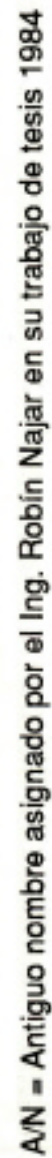




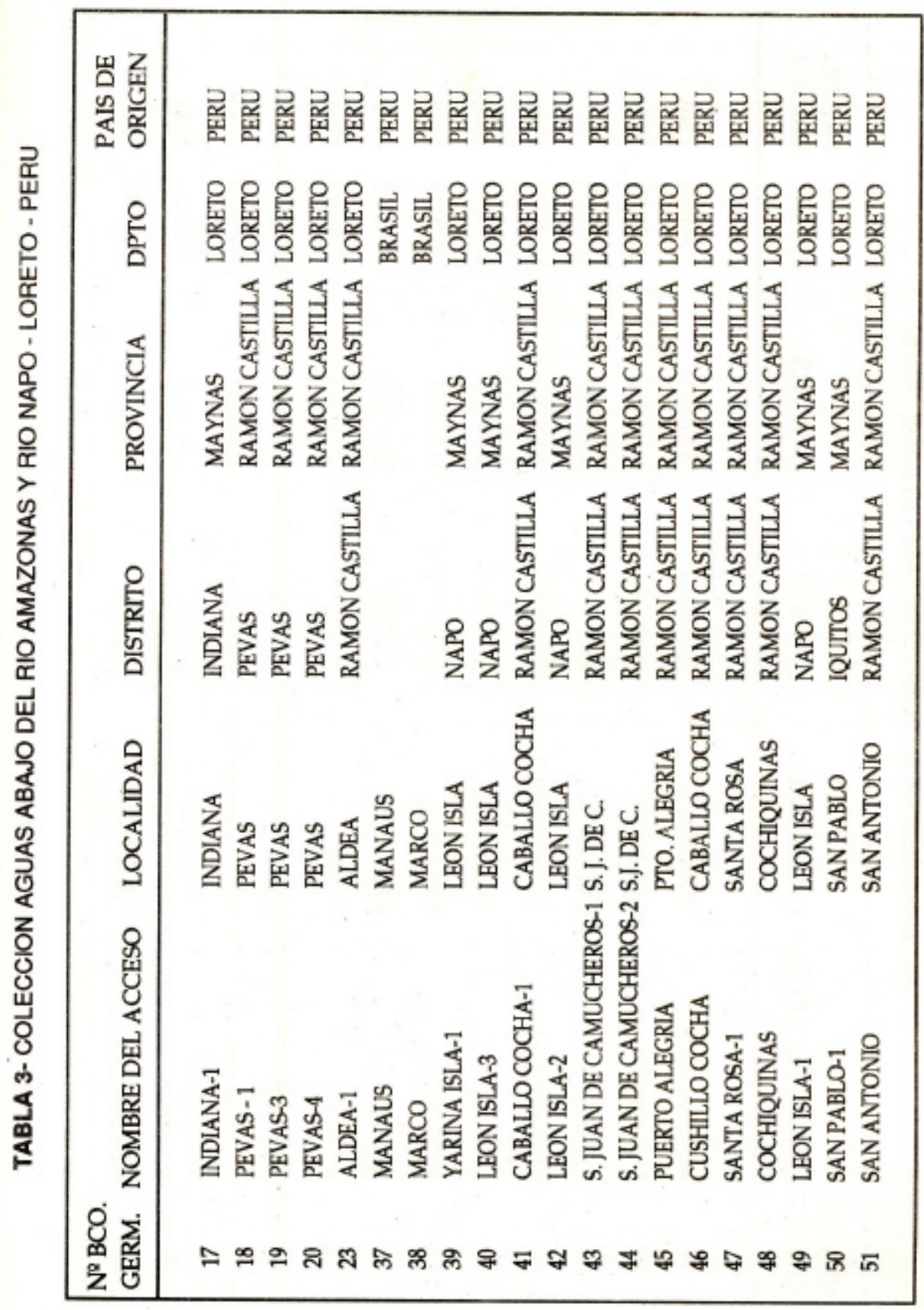




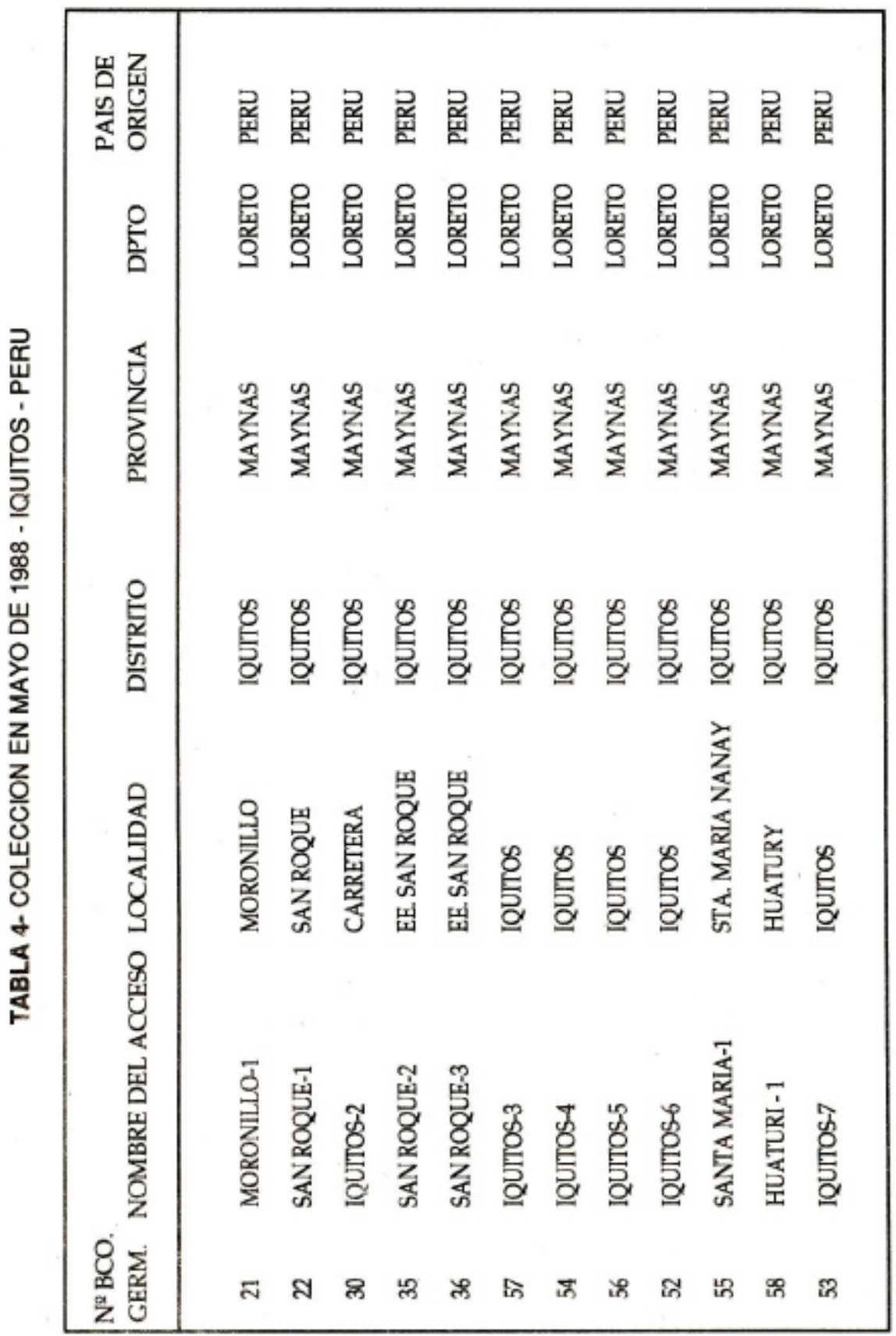




\section{RESULTADOS}

\subsection{Colecciones}

3.1.1 Colecciones en el Banco de Germoplasma de achiote de la Estación Experimental "EL TULUMAYO", Tingo María, Provincia de L eoncio Prado, D pto. de Huánuco - Perú.

En total se colectaron 17 accesos, de las cuales 06 procedían del Centro Agronómico Tropical de Investigación y Enseñanza (CATIE)- Costa Rica, las restantes proceden de los dptos. De Loreto, U cayali, Huánuco, Junin. Estas colecciones se realizaron en setiembre de 1986, y se describen en la tabla 1.

3.1.2 Colecciones de accesos de achiote en el campo experimental ALLPAHUAYO; esta plantación (que se mantiene), fue instalada por Nájar (1984) durante su trabajo de tesis, los ecotipos existentes, fueron incluidos en el banco de germoplasma en agosto de 1987.

Estas colecciones originalmente fueron colectadas en Setiembre de 1983 en los Dptos. De Loreto y U cayali, sembrados en una área cercana al campo experimental Allpahuayo. Los detalles de colección se presentan en la tabla 2.

3.1.3 Colecciones aguas abajo del origen del rió A mazonas y el rió $\mathrm{N}$ apo. Estas colecciones se relazaron en junio de 1987, se incluyeron en el Banco de Germoplasma en abril de 1988, los detalles se describen en la tabla 3.

3.1.4 Colecciones locales. Estas colecciones se efectuaron dentro de la jurisdicción del distrito de Iquitos, las que comprenden caseríos cercanos a la ciudad de lquitos, fueron incluidos en el Banco de Germoplasma, en mayo de 1988, en la tabla 4 se describen las localidades de las colecciones.

\subsection{Mantenimiento}

Para fines de mantenimiento de las colecciones realizadas, se eligió un área de terreno en el campo experimental Allpahuayo, del Instituto de Investigaciones de la A mazonía Peruana (IIAP), situado en el Km. 20.5 de la 
Carretera Iquitos - Nauta. El banco de germoplasma de achiote actualmente cuenta con 69 accesos, que se mantienen "in vivo" sembrados con una densidad de $5 \times 4 \mathrm{~m}$.

Cada acceso cuenta con un máximo de 10 plantas y un mínimo de 7, el número menor se debe a muerte de individuos después de sembrados en el campo definitivo.

\subsection{Variabilidad de la Especie}

De acuerdo a las fichas de las colecciones realizadas y los descriptores utilizados, se puede encontrar una gran variabilidad referida a:

- Hábito de crecimiento. Podemos encontrar individuos herbáceos, así como plantas de hábito arbóreo.

- Forma de la copa de la planta; se puede encontrar hasta 3 formas; ovoide, hemisférica y cónica.

- Coloración del tallo; se encontró colores anaranjados, marrones, grises con moteaduras.

- Coloración de las flores; las flores encontradas desde blancos, lilas, rosadas y violetas.

- Visibilidad de las panículas; respecto a esta característica se pudo encontrar panículas con un alto grado de exposición.

- La alta variabilidad con respecto al fruto se pudo observar en los siguientes: coloración (verde, rojo, amarillo, negro y marrón); forma (ovoide, redondo, elíptico y cónico); espinosidad (sin espinas, muy baja, baja, al ta y muy alta); longitud de las espinas (muy cortas, cortas, largas y muy largas).

\section{DISCUSION}

Las áreas de distribución en la A mazonía Peruana se ubican en al cuenca del Amazonas con sustributarios en el departamento de Loreto (Hernández 1988), sin embargo la mayor producción nacional y superficie cultivada se encuentra en los departamentos de Cuzco y A yacucho (FOPEX) dado que en la Selva Baja solo se realiza una actividad de recolección de semillas.

El A chiote en la selva peruana son plantas de hábitos arbustivos y arbóreos; según Cevallos 1978, es una planta de porte bajo $3-4 \mathrm{~m}$. (arbustivo), mientras 
León 1968, es una planta que puede llegar excepcionalmente hasta $10 \mathrm{~m}$. (arbóreos).

La diversidad genética de la especie es muy amplia, referida a características fenotípicas. Hernández 1988. En la selva peruana el achiote presenta una heterogenidad de sus características botánicas tales como tamaño y forma de la planta, así como forma y color de sus hojas, flores y frutos.

\section{CONCLUSIONES}

-En la A mazonía Peruana existen gran diversidad genética de la especie Bixa orellana L. "achiote".

-Existe un gran potencial de desarrollo del cultivo del achiote en la selva baja, por ser una especie adaptada a las condiciones agroecológicas de la zona.

\section{BIBLIOGRAFIA}

CATALAN, Z. 1974. A chiote. Información para Técnicos, M inisterio de A gricultura, Guatemala C.A. 13 p.

FONDO DE PROMOCION DE EXPORTACIONES NO TRADICIONALES Achiote Bixina. FOPEX - Lima. Perú S/F.

HERNANDEZ, T. et al. 1988. Sistemas de Producción de Achiote en la amazonía peruana. CORDEHUANUCO, Tingo María - Perú, 29p.

HOLDRIGDE, L. R. 1959. Ecología Tropical of used for N ew A presh to Tropical Land USA. Economic Botany. $271 \mathrm{p}$.

INSTITUTO DE INVESTIGACIONES DE LA AMAZONIA PERUANA 1986. Proyecto Recursos Fitogenéticos de la A mazonía, IIAP - Iquitos, Perú 29p.

INSTITUTO DE INVESTIGACIONES DE LA AMAZONIA PERUANAI987.Estudios de recursos Fitogenéticos, Alimenticios e Industriales, Informe Técnico 1986. IIA P - Iquitos, Perú.

LEON J. 1968 Fundamentos Botánicos de los cultivos tropicales. Instituto Interamericano de Ciencias A grícolas de la OEA. San José., Costa Rica. 437 pág. 
NAJAR, G.R.E. 1984. Evaluación del contenido de Bixina en Cultivares de Achiote (Bixa orellana L.) Tesis A gronomía - UNAP - Iquitos. 162p.

OCAMPO, 5. 1983. A spectos A gronómicos sobre el cultivo del A chiote (Bixa orellana L.) en los Cantos es de Aguirre y Danata. In. Aspectos sobre el Achiote y perspectivas para Costa Rica. Ed. Jorge Arce P. CA TIE - Turrialba. COSTA RICA . pp. 43, -57.

RODRIGUEZ B y G. ENRIQUEZ 1983. Estudio Preliminar del Desarrollo de Ramas y Biología floral en Bixa orellanaL. In. A spectos sobre el achiote y perspectivas para Costa Rica. Ed. Jorge A rce P. CA TIE, Turrialba, Costa Rica .pp.58y 76. 